\title{
A Microanalytical Device for the Assessment of Coagulation Parameters in Whole Blood
}

\author{
Celine Bisson, John Campbell, Rhonda Cheadle, Marianne Chomiak, John Lee, Cary Miller, Catherine \\ Milley, Peter Pialis, Sandra Shaw, Wenda Weiss, Cindra Widrig \\ i-STAT Canada Ltd. \\ Kanata, Ontario K2L 1T9
}

\begin{abstract}
The following describes the development of a disposable cartridge for use at the patient side to perform traditional coagulation assays on fresh whole blood samples. The cartridge provides a means by which a blood sample can be metered and quantitatively mixed with reagents which activate the coagulation cascade. Clot formation is subsequently detected using a microfabricated sensor also housed within the cartridge. The functional features of the cartridge and sensor are described and clinical results are presented.
\end{abstract}

\section{INTRODUCTION}

i-STAT Corporation manufactures a system for patientside testing comprised of disposable test cartridges and a hand held or patient-monitor-contained analyzer. The cartridge is assembled from molded components and houses the necessary calibrants and sensors to perform a variety of clinical chemistry tests. Currently i-STAT produces cartridges containing various combinations of electrochemical sensors which have been miniaturized through microfabrication techniques. Thin-film photolithographic processes and nanoliter partial-drop dispensing technology are utilized to produce potentiometric $\left(\mathrm{Na}^{+}, \mathrm{K}^{-}, \mathrm{Cl}^{-}\right.$, urea, $\mathrm{Ca}^{+2}, \mathrm{pH}$, and $\left.\mathrm{CO}_{2}\right)$, amperometric (glucose, creatinine, oxygen), and conductometric (hematocrit) sensors. iSTAT manufactures 10 products containing various combinations of these 11 tests. The sensors are packaged so that single cartridges accommodate the most common testing patterns.

A typical cartridge is shown in Figure 1. Conduits and valves used for calibrant and sample delivery are indicated. The biosensors reside on the chips at the top of the cartridge. The reagents needed to carry out the signal generating reaction on each sensor are contained within layers at the sensor's surface. This eliminates chemical cross-talk and allows the tests to be performed in a single conduit. No accurate fluid positioning is required.

Our R\&D plan intends the addition of several new classes of tests to the currently available battery so that complete patient-side testing is available in a single instrument. To perform these tests, new functionality will be added to the cartridge and features within the present analyzer that are not yet employed in current product will be put to use. Future tests will be run on today's platform, however, and future cartridges will be manufactured with modifications of current processes.

Under development at present is a series of new cartridges designed to perform coagulation assays in existing
i-STAT instruments. These tests are used to assess the function of an individual's hemostatic system. In an assay, the enzymes responsible for coagulation are activated by the addition of specific reagents and the elapsed time to clot formation is measured.

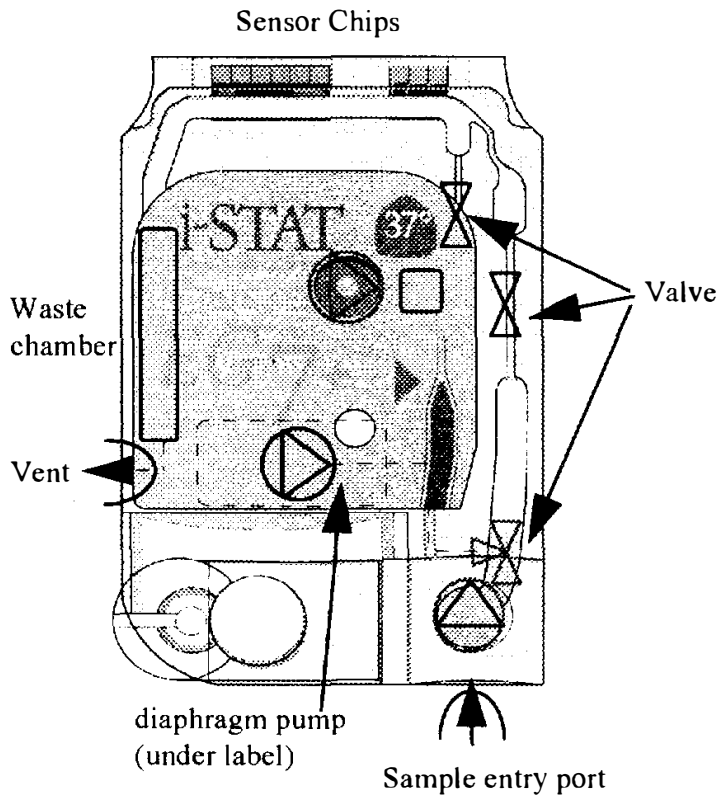

Figure 1. i-STAT cartridge for electrolyte and blood gas testing. Valves and pumps are schematically indicated.

\section{BACKGROUND}

Coagulation in blood or plasma occurs when fibrinogen is enzymatically converted to fibrin. In this conversion, small peptide fragments are cut from the fibrinogen molecule to produce individual fibrin strands. The strands then forn a hydrogen-bonded network that serves to gel the sample. The enzyme responsible for liberation of the fibrinopeptides is the protease thrombin. It is generated in its active form as the penultimate step in the "coagulation cascade" -- a series of sequential protease activations involving nine plasma proteins. This cascade is depicted in Figure 2.

The most common coagulation tests are performed in platelet free plasma that is taken from centrifuged whole blood. During collection, trisodium citrate is added to the blood to temporarily remove ionized calcium, a required cofactor for a number of the protease reactions. This prevents clotting during transport and handling of the sample. A test is perfonned by adding an activator to the plasma, incubating the mixture to allow complete activation of the first enzyme in the cascade, and finally adding calcium chloride in excess of the citrate 


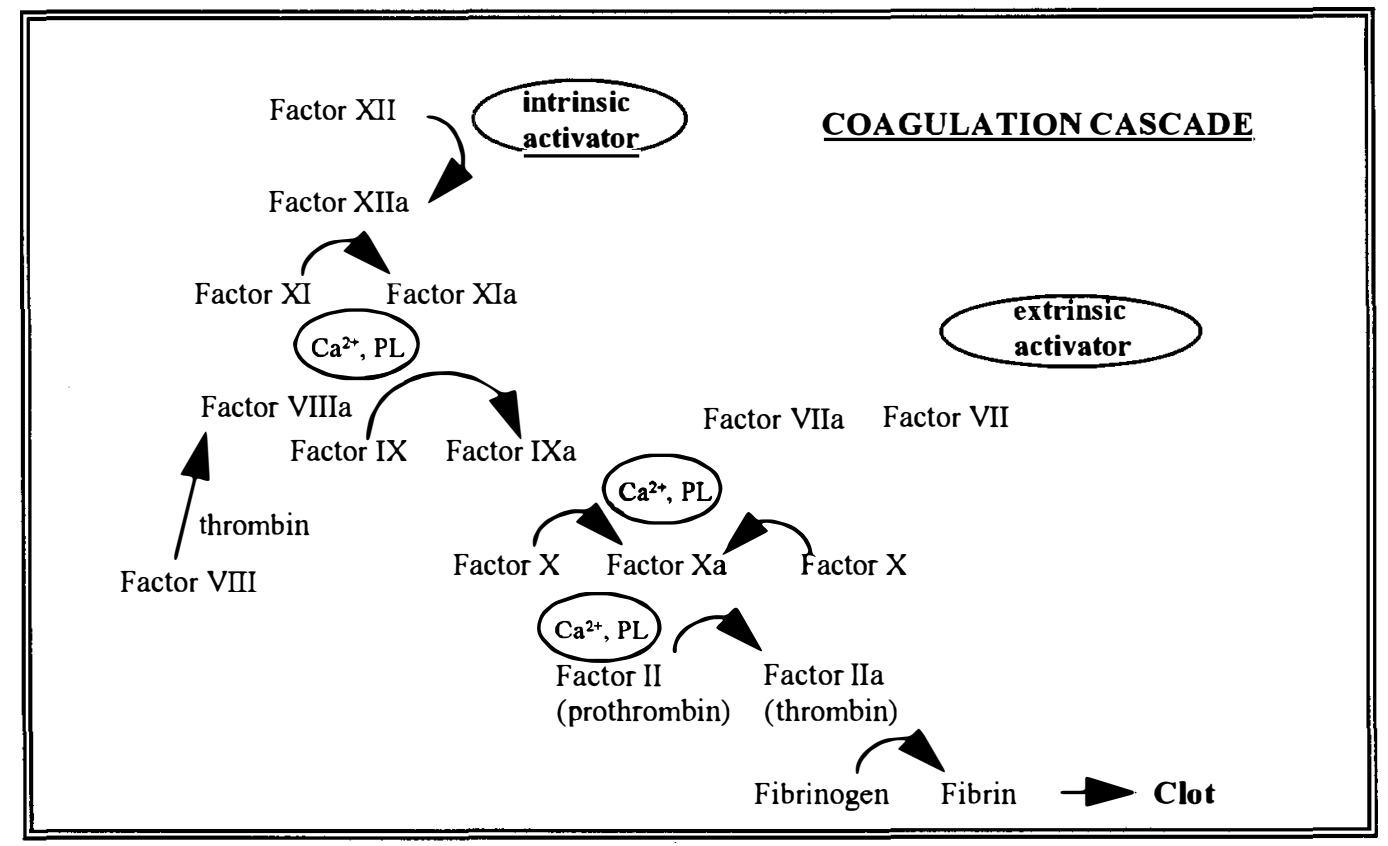

Figure 2. Enzymatic steps occurring in the coagulation cascade. Each factor shown is present in plasma as an inactive protease. During coagulation, the enzymes are sequentially converted to their active forms, denoted by the appended " $a$ ". The arrows indicate the sequence of activations required for clot formation. As indicated, many of the reactions also require the presence of free calcium ions and phospholipids (PL). Coagulation can be initiated through the activation of the intrinsic (activation of Factor XII) or extrinsic pathway (activation of FactorVII).

concentration to allow coagulation to proceed. The endpoint is reached when clot formation is detected either optically (as the plasma becomes turbid) or mechanically (as the plasma viscosity increases) !. A Prothrombin Time test (PT) adds an extrinsic activator and measures the time for clot formation via activation of Factors VII, X, and II. An activated Partial Thromboplastin Time test (aPTT) initiates coagulation with an intrinsic activator and is used to assess the function of Factors XII, XI, DX, VIII, X, and II. It is also sensitive to inhibitors of these enzymes.

The functions which must therefore be incorporated within the cartridge are a means by which reagent may be quantitatively mixed into a blood sample and a method for detecting a clot. These two aspects of the cartridge design are discussed below.

\section{ADDITION OF REAGENT TO A METERED BLOOD SEGMENT}

To initiate coagulation, reagent must be rapidly and quantitatively mixed into a test sample. In a benchtop instrument, this is accomplished by injecting an aqueous solution containing the reagents into a measured aliquot of plasma. Although it is possible to engineer similar "stop-flow" mixing into a cartridge, it has been found that the simpler dissolution of dry reagent provides adequate speed and reproducibility of homogenization.

Current single test devices which do not have convective mixing capability typically employ a high surface-area-tovolume ratio reaction vessel in which the walls of the cell are coated with dry reagent. Diffusion provides sufficiently rapid homogenization. As the required dissolution time in the coagulation tests is on the order of seconds, the upper limit of such a chamber's shortest dimension is approximately 25 microns. Such geometries, however, do not easily lend themselves to a multi-assay format or to the integration of noncoagulation tests. Because it is intended that the mixing strategies devised here be generally applicable to future cartridges types, larger bore conduits allowing serial testing and fluid wash-out are preferred. With larger diameter channels, convection is required to provide rapid mixing.

In the coagulation cartridge, the clot reaction is initiated in a specific region of the flow channel over the sensor chips. A length of the wall within the channel is coated with reagent, as indicated in Figure 3. In other applications, reagent may be added in the pre-sensor channel or sample holding chamber. Convection is induced by oscillating a segment of blood in the region of the flow channel containing the reagent. The motion is controlled so that the trailing edge of the blood segment continually moves on and off of the reagent coating, as depicted in Figure 4. This mixing profile confines the dissolving reagent to a defined region of the blood. The remainder of the segment is available for the addition of other reagents or testing with other sensors.

The oscillation is maintained with feedback from the fluid sensor coincident with the reagent coating. This sensor comprises the two parallel bars on the chip shown in Figure 5. These bars lie perpendicular to the length of the flow channel and the electrical resistance between them can be used to monitor the relative position of the fluid front. Feedback from this sensor is used to control the motor direction and speed so that the center, amplitude, and frequency of the oscillation can be maintained. With such control it is possible to obtain 
reproducible mixing profiles in varying hematocrit (and therefore viscosity) samples.

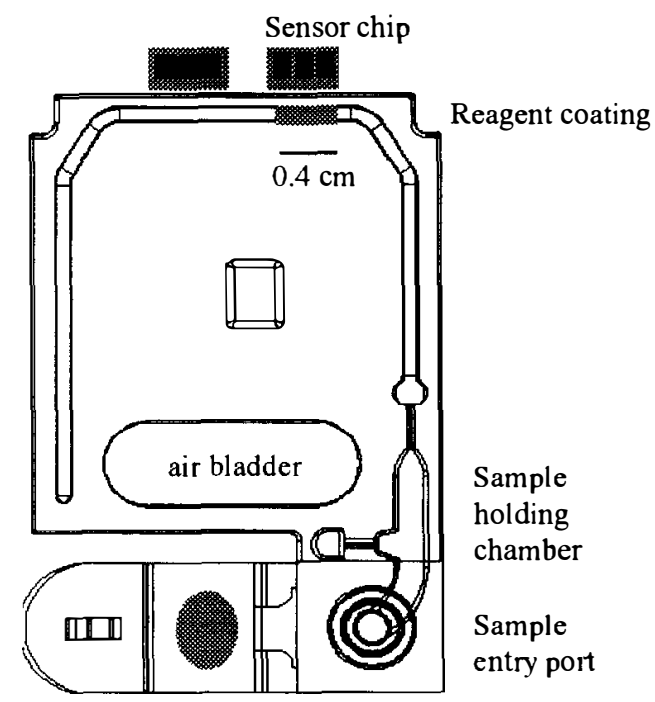

Figure 3. Coagulation Cartridge with air bladder and reagent coating shown. Sample holding chamber is approximately 25 microliters. The forward 10 microliters of sample is moved into the sensor channel for the coagulation assay. The upper right portion of the sensor channel is coated with a reagent layer. The sensor chip coincident with this segment contains the endpoint sensor and the fluid positioning sensor. It is the chip shown in Figure 5.

Figure 6 shows the resulting concentration gradient along the channel. The sample used here is whole blood. In this experiment, an aqueous solution containing an electroactive marker was deposited in the region of the flow channel indicated above and dried. Complete dissolution of the reagent into a $0.4 \mathrm{~cm}$ section of blood would lead to a concentration in that segment of $80 \mu \mathrm{M}$. The graph indicates the oxidation current after mixing. It also shows the current expected in unadulterated blood and that containing $80 \mu \mathrm{M}$ of the marker. The individual points represent the current at an electrode in the position indicated by the $x$ axis. Across the $2 \mathrm{~mm}$ of solution at the edge of the sample, the concentration varies by approximately $10 \%$.

Once mixing has been completed, the segment of the blood containing the reagent must be held above the sensing electrode. This is also accomplished using the signal from the conductometric sensor to maintain a specified resistance typical of the fluid front resting between the two electrodes. In this manner the fluid position along the channel can be controlled to within $0.1 \mathrm{~mm}$.
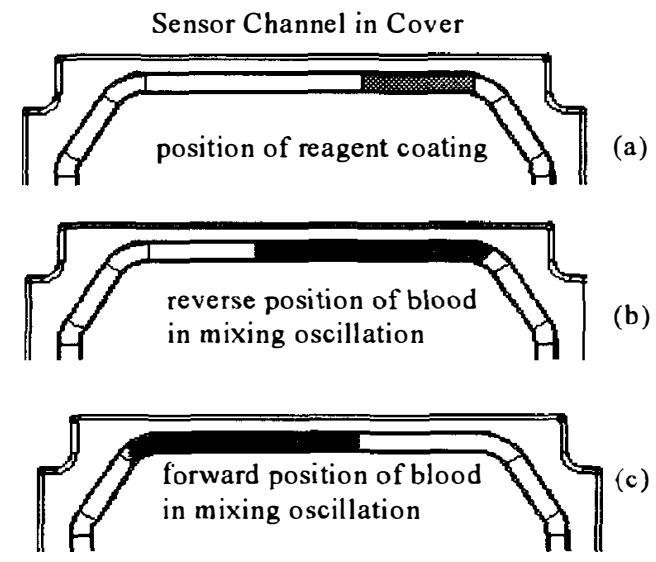

Figure 4. Position of blood during oscillation for dissolution of reagent coating the flow channel as depicted in (a).

\section{CLOT DETECTION}

The preferred detection methodology at i-STAT is that which results in the lowest cost for integration of multiple tests into a single platform. This has lead to the development of electrochemical sensors, of which there are several options available for coagulation testing. Below are examples of viscometric and electrogenic endpoint detection schemes which may be used.

Viscometric Endpoint Detection. The relative viscosity of a sample can be assessed by monitoring the velocity of a column of that fluid as a function of the pressure differential across the length of the segment. For a constant pressure profile, increasing viscosity will be manifested as a slowing in the fluid. To make an electrochemical viscometric detector in this cartridge, a means of imparting a pressure gradient and an electrochemical detector of the fluid speed are required.

The former is achieved by oscillating the fluid via the air bladder as described above. This produces a roughly sinusoidal variation in the pressure gradient with an amplitude and frequency determined by the plunger motion. The fluid speed can be monitored using amperometric, conductometric, or potentiometric sensors. For example, if there is an electroactive species in the oscillating fluid, the current for its reduction (or oxidation) at a disc electrode will also oscillate. It increases as the stagnant layer at the electrode surface thins during acceleration and decreases as the depletion layer grows when the fluid stops to turn around. The AC and DC components of this signal can be tailored by adjusting the electrode geometry and fluid motion profile.

Once the blood begins to clot, the increased viscosity lessens the response to the pressurization. Both the amplitude of the AC signal and the DC offset decrease. A clot curve is shown in Figure 7. Events within the curve, such as a shift in the phase angle of the AC current relative to plunger motion, may be used to indicate the coagulation endpoint. 


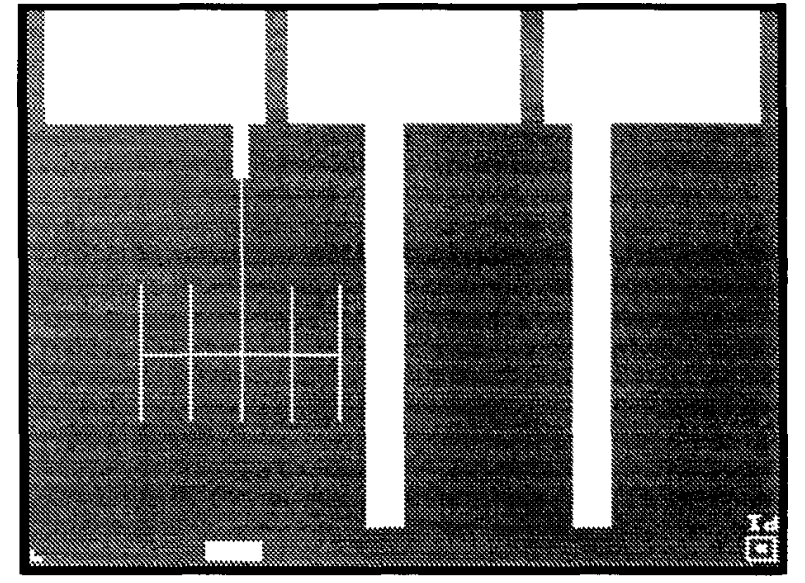

Figure 5. Sensors for fluid positioning and amperometric detection. The two bars on the right together make up the conductometric sensor. Their output is a measure of the local channel resistance. They are used to monitor the position of the fluid edge during mixing and to maintain the position of this edge throughout testing. The sensor to the left is an that used endpoint detection. For reference, the contact pads shown at the top of the chip are $1 \mathrm{~mm}$ wide.

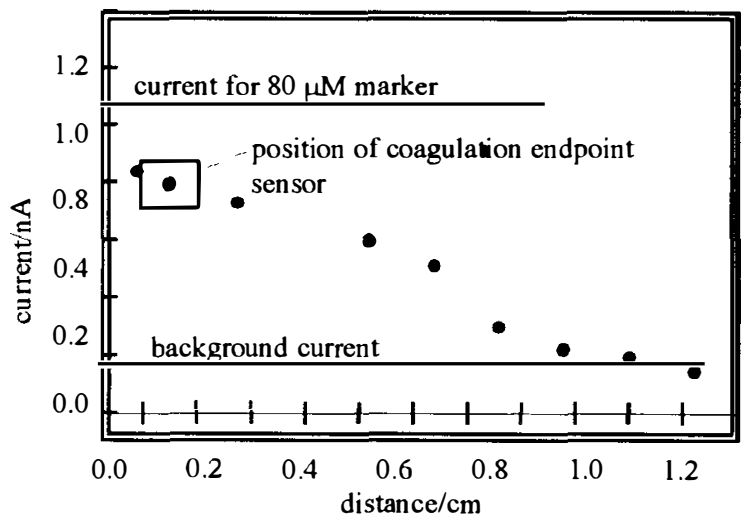

Figure 6. Resulting current for the described dissolution of a typical electroactive marker in blood. The measurements were made with sensors downstream of the conductometric sensor by the distance on the $x$ axis. If dissolved completely in $0.4 \mathrm{~cm}$ of the channel, the quantity of the marker present would result in an $80 \mu \mathrm{M}$ solution.

The sensing electrode used in this assay merits note. The specification is that it must provide a means for direct oxidation or reduction of a species dissolved in whole blood. It also must remain stable for up to 15 minutes, the longest a clot test will run. This means that the surface of the electrode must be protected against fouling from blood components yet remain accessible for direct electron transfer by the redox species. Thick semi-permeable protective coatings are not satisfactory, however, as they would not provide the necessary sensitivity to fluid motion. To meet these criteria, electrode coatings deposited using molecular self-assembly and/or plasma processing techniques have been developed.

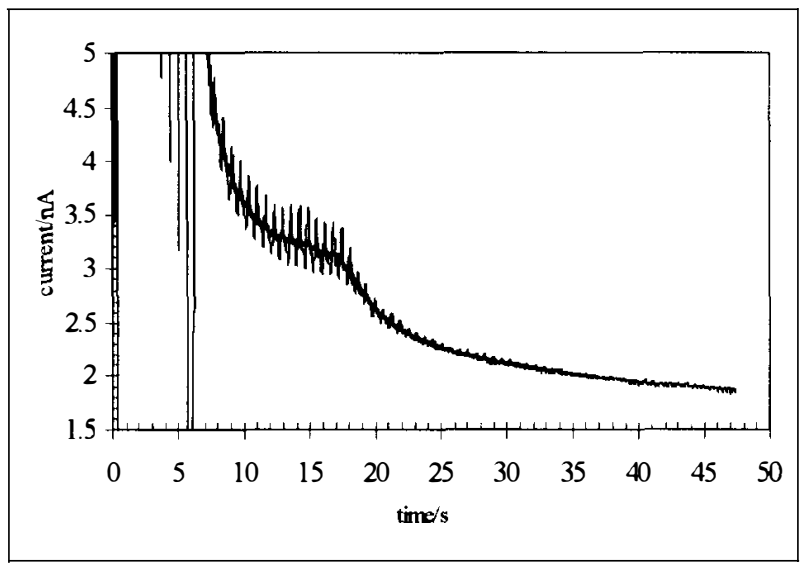

Figure 7. Electrochemical viscometric clot detection in a PT test. Shoun is the current for the oxidation of an electroactive marker used as an indicator of clot formation. the marker is mixed into the blood along with activators of the intrinisic coagulation pathway. The high current from 0 to 5 seconds occurs as a result of the relatively rapid fluid motion during mixing. The oscillating current between approximately 8 and 17 seconds is as expected for the low amplitude oscillation of un-clotted blood. As the viscosity increases due to clotting, the $A C$ level and $D C$ offset of the signal both decrease.

Electrogenic Endpoint Detection. Fibrinogen is cleaved by activated thrombin. The time at which this enzyme is produced in the active form consequently correlates to the time for physical clot formation. The ability to detect thrombin therefore offers an alternative method of endpoint detection.

Thrombin is a protease that hydrolyzes peptides at the carboxyl terminal of arginine. Its presence can therefore be determined by the addition of an arginine containing substrate which, upon conversion, generates a colored, fluorescent, or electroactive species. A number of such substrates have been described in the literature ${ }^{2}$ and chromogenic endpoint detection has been used to determine clot times in plasma samples ${ }^{3}$.

An electrode like the one described above can be used to amperometrically detect the liberated electrophore directly. Alternatively, the signal may be carried to the sensing electrode via mediators which have chemically oxidized or reduced the generated marker. The latter strategy offers several advantages. For example, it removes the need for an electrophore with rapid heterogeneous electron transfer kinetics. It also allows the use of "thick" electrode coatings which will diminish noise induced by incidental fluid motion. Furthermore, a mediator couple may also be detected potentiometrically. The latter point is particularly relevant for design flexibility of multiple-test cartridges. Both amperometric and potentiometric sensors for endpoint detection have been developed. Clot curves resulting from the conversion a substrate are shown in Figure 8. Again, a number of features from these curves may be used to indicate the endpoint. 


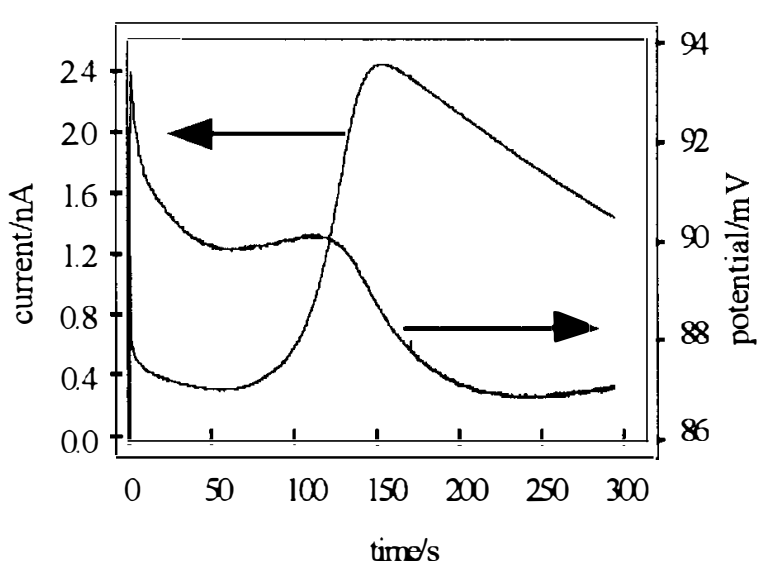

Figure 8. Electrogenic Clot Curve. The electrogenic substrate along with activators of the intrinsic pathway are mixed into a blood sample prior to the time marked as 0 . The signals shown are the amperometric (left axis) and potentiometric (right axis) detection of the electroactive marker liberated by thrombin. The current increases (potential decreases) when thrombin is generated and plateaus once the substrate is completely hydrolyzed.

Combination Method. In that the most common clot detection methods rely on the formation of a physical clot, these tests are sensitive to deficiencies in any of the coagulation proenzymes as well as deficiencies in fibrinogen. Because the electrogenic method determines only the time for the activation of thrombin, however, it will not be prolonged in the event of a low fibrinogen sample. To make an electrogenic endpoint "match" a viscometric test one therefore needs an estimate of the sample fibrinogen to correct the thrombin time when necessary

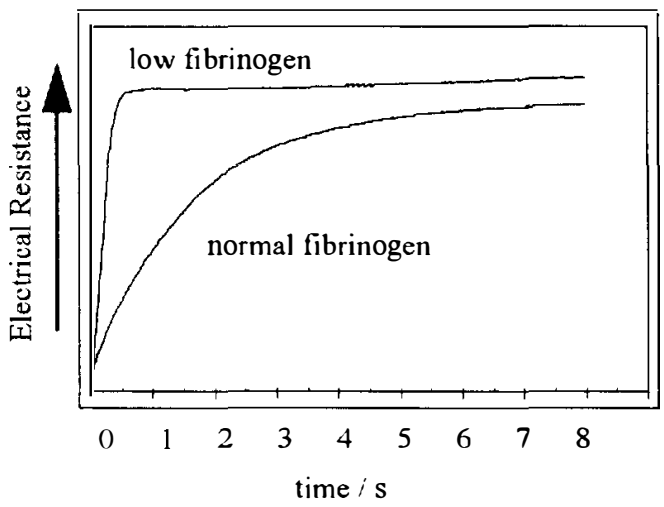

Figure 9. I'iscometric assessment of the clotted sample. At $t=0$ the air bladder is compressed to induce movement in the clotted blood. As the fluid is pushed off of the conductometric sensor, the measured electrical resistance between its electrodes increases. The rate of change of the resistance is indicative of the speed of the moving fluid and therefore the fibrin content of the clot.

Because the flow resistance of a clotted sample increases with increasing fibrin concentration, a measurement of this resistance can be made to assess the concentration of fibrinogen originally present in the sample. This determination is made by gently pushing the clotted sample and measuring the time transient for the resulting fluid motion. Again the conductometric sensor is used to monitor the fluid response. Electrical resistance transients for samples with low and normal fibrinogen concentrations are shown.

\section{TEST RESULTS}

To be accepted, this test must have the same anticoagulant and factor sensitivities as the commonly used methods. It also must be similarly precise. The former is demonstrated in method comparison studies while the latter is ascertained through the use of commercially available control plasmas. Some results are presented below.

Precision. Precision is most easily assessed using commercially available control plasmas. Table 1 shows the clot time, within sample precision, sample to sample precision, and total precision for i-STAT aPTT cartridges as well as the reference benchtop instrument. The samples were prepared by re-hydrating lyophilized control plasmas with de-ionized water as per the manufacturer's instructions and re-calcifying to normal levels with $25 \mathrm{mM} \mathrm{CaCl}_{2}$. The total precision of the new and reference tests is comparable.

Table 1

Precision data for normal level control plasmas in

\begin{tabular}{|c|c|c|}
\hline Parameter & $\begin{array}{c}\text { i-STAT } \\
\text { aPTT } \\
\text { Cartridges }\end{array}$ & $\begin{array}{c}\text { MLA 700 } \\
\text { w/ Pacific } \\
\text { Hemostasis } \\
\text { Kontact Reagent }\end{array}$ \\
\hline result & 28.6 seconds & 27.4 seconds \\
\hline number of test events & 15 & 22 \\
\hline samples per test event & 2 & 2 \\
\hline within run precision & 0.73 seconds & 0.91 seconds \\
\hline run to run precision & 0.71 seconds & 0.83 seconds \\
\hline total precision & 1.02 seconds & 1.23 seconds \\
\cline { 2 - 3 } & \multicolumn{2}{c|}{}
\end{tabular}

Anticoagulant sensitivity. The Activated Clot Time (ACT) test is the standard test used to determine the adequacy of anticoagulation during cardiovascular surgery and renal dialysis. It is traditionally a whole blood test in which an activator of the intrinsic pathway is added to the samples and the time to physical clot formation, measured viscometrically, is determined. It is used to monitor heparin, an anticoagulant, at high doses. Figure 10 shows the correlation of the i-STAT cartridge to measurements made on an Array Medical Actalyke reference instrument. Samples were prepared by spiking freshly drawn whole blood with sodium heparin to create concentrations from 1 to $5 \mathrm{U} / \mathrm{ml}$ plasma. 


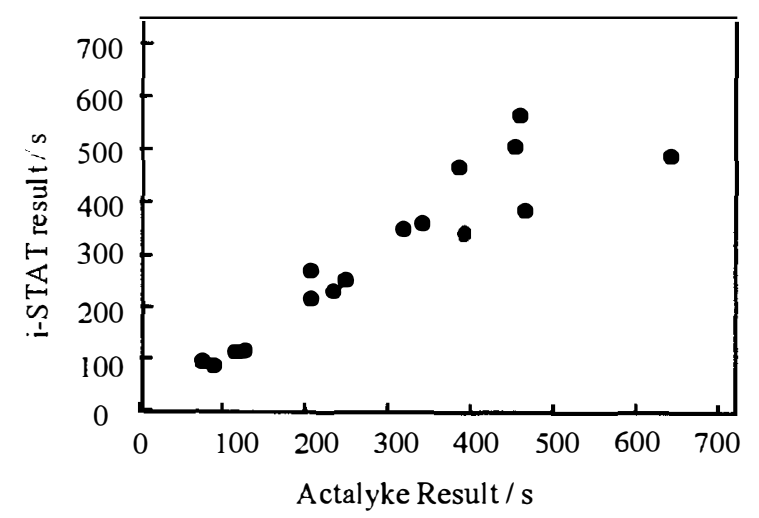

Figure 10. Coagulation times in an Activated Clot Time test (ACT) vs. a reference instrument.

Factor Sensitivity. Factor deficient citrated plasma samples were mixed in varying proportions with normal plasma to produce samples with partial factor deficiencies. The plasma was then mixed with washed red blood cells and re-calcified to produce factor deficient whole blood samples. The blood samples were run in test cartridges and the plasma samples were tested on a benchtop plasma instrument (MLA 700 using Pacific Hemostasis Kontact Reagent). The results are shown in Table 1. The values given are quotients of the clot times for the abnormal and normal samples. Table 1 also shows similar data obtained using a reference whole blood instrument.

Table 2

\section{Factor sensitivity of the aPTT test}

\begin{tabular}{|c|c|c|c|c|}
\hline Sample & $\begin{array}{c}\% \% \\
\text { Factor } \\
\text { Present }\end{array}$ & $\begin{array}{l}\text { Plasma } \\
\text { aPTT } \\
\text { ratio for } \\
\text { i-STAT }\end{array}$ & $\begin{array}{c}\text { Plasma } \\
\text { aPTT } \\
\text { ratio } \\
\text { for } \\
\text { MLA* }\end{array}$ & $\begin{array}{c}\text { Plasma } \\
\text { ratio for } \\
\text { Coagu- } \\
\text { Check Plus } \\
\text { cartridge }\end{array}$ \\
\hline Normal & 100 & 1.00 & 1.00 & 1.00 \\
\hline \multirow[t]{4}{*}{ Factor VIII } & 50 & 1.12 & 1.15 & 1.55 \\
\hline & 25 & 1.19 & 1.05 & \\
\hline & 10 & 1.41 & 1.35 & 2.52 \\
\hline & 5 & 1.63 & 1.47 & \\
\hline \multirow[t]{4}{*}{ Factor IX } & 50 & 1.12 & 1.12 & 1.16 \\
\hline & 25 & 1.23 & 1.27 & \\
\hline & 10 & 1.35 & 1.44 & 1.76 \\
\hline & 5 & 1.57 & 1.59 & \\
\hline \multirow[t]{4}{*}{ Factor X } & 50 & 1.12 & 1.23 & \\
\hline & 25 & 1.14 & 1.3 & \\
\hline & 10 & 1.3 & 1.43 & \\
\hline & 5 & 1.42 & 1.64 & \\
\hline \multirow[t]{4}{*}{ Factor XI } & 50 & 1.04 & 0.97 & 1.45 \\
\hline & 25 & 1.69 & 1.52 & \\
\hline & 10 & 1.02 & 1.69 & 2.38 \\
\hline & 5 & 2.24 & 2.23 & \\
\hline \multirow[t]{4}{*}{ Factor XП } & 50 & 1.46 & 1.18 & \\
\hline & 25 & 1.72 & 1.21 & 1.13 \\
\hline & 10 & 1.94 & 1.32 & \\
\hline & 5 & 2.34 & 1.45 & 1.85 \\
\hline
\end{tabular}

* In-house results using citrates plasma ** Results taken from CoaguCheck Plus package insert

\section{CONCLUSION}

Cartridges for performing coagulation assays using the i-STAT platform has been developed. The cartridge performance equivalent to that of the benchtop plasma instruments and other point-of-care whole blood analyzers.

${ }^{1}$ Machin, S.J.; Mackie, I.J.; Chitolie, A.; Lawrie, A.S. Clin. Lab. Haem., 1996, 18, 1-6

${ }^{2}$ Claeson, Blood Coagulation and Fibrinolysis, 1994, 5, 411 :: Peuriot,M.; (b) Nigretto, J-M.; Jozefowicz, M. Thrombosis Research, 1981, 22, 303.

${ }^{3}$ Walenga, J.; Fareed, ZJ. Bermes, E.W., Seminars in Thrombosis and Hemostasis, 1983, 9, 172. (b) Becker, U., Bartl, K.; Lill, H.; Wahlefeld, W., Thrombosis Research, 1985, 40, 721 